\title{
Validação de construto e consistência interna do IMPRAFE-54 em atletas de basquetebol infantojuvenis
}

http://dx.doi.org/10.11606/1807-5509201900030467

\author{
Ricardo Pedrozo SALDANHA* \\ Gabriel Henrique Treter GONÇALVES ${ }^{* *}$ \\ Marcus Levi Lopes BARBOSA*** \\ Nígia Ramalho ARSEGO** \\ Marcos Alencar Abaide BALBINOTTI**** \\ Carlos Adelar Abaide BALBINOTTI**
}

${ }^{*}$ Centro Universitário La Salle, Canoas, RS, Brasil.

**Universidade Federal do Rio Grande do Sul, Porto Alegre, RS, Brasil.

***Universidade Feevale, Novo Hamburgo, RS, Brasil.

****Université du Québec à Trois-Rivières, Trois-Revières, Québec, Canadá.

0 presente estudo teve por objetivo apresentar novas evidências de validade e precisão do Inventário de Motivos à Prática Regular de Atividades Físicas e/ou Esportivas (IMPRAFE-54) com atletas de basquetebol infanto-juvenis. Participaram do estudo 438 jovens atletas de basquetebol de ambos os sexos, com idades entre 13 e 16 anos, vinculados a instituições esportivas. Foram respondidos dois instrumentos: 0 Questionário de Identificação e Controle de Variável (QICV) e o Inventário de Motivos à Prática Regular de Atividades Físicas e/ou Esportivas (IMPRAFE-54). Foi realizada uma estimação da estrutura interna do construto segundo os dados disponiveis a título de verificação prévia do modelo segundo os pressupostos teóricos, a qual confirmou o modelo hexadimensional. As seis dimensões apresentaram índices Alpha de Cronbach superiores a 0,86, tratando-se, portanto, de um inventário válido e fidedigno. Para testar se o modelo exploratório se confirma foi calculada a análise fatorial confirmatória ULS (Unweighted Least Squares) e seus resultados $\left(x^{2}=4723,807 ; g l=1362 ; p<0,001 ; x^{2} / g l=3,468 ; \mathrm{GFI}=0,970 ; \mathrm{AGFI}\right.$ $=0,967 ; \mathrm{SRMR}=0,0574 ; \mathrm{NFI}=0,962)$ se mostraram aceitáveis. Este estudo, portanto, indica que 0 IMPRAFE-54 é aplicável a uma população específica, no caso jovens atletas de basquetebol participantes de competições institucionalizadas. Estes resultados podem ser particularmente úteis para treinadores e psicólogos do esporte, visto que a partir da análise dos motivos que levam estes indivíduos à prática do basquete, podem planejar de forma mais adequada seus treinamentos e intervenções.

Palavras-chave: Motivos; Psicometria; Validação; Esporte.

\section{Introdução}

Os motivos podem ser entendidos como razóes pelas quais as pessoas se engajam em uma determinada atividade. Todos os motivos são sustentados por metas do sujeito, que são subjacentes a estes ${ }^{1}$. No caso do sujeito que afirma que seu motivo para a prática da atividade física é "fazer novos amigos", tem como meta melhorar seu nível de sociabilidade. A literatura tem mostrado que os principais motivos que levam as pessoas à prática da atividade física são: Controle de Estresse, Saúde, Sociabilidade, Competitividade, Estética e Prazer ${ }^{2,3}$. O Inventário de Motivos à Prática Regular de Atividade Física e/ou Esportiva (IMPRAFE-54) ${ }^{4}$ é um instrumento que viabiliza o acesso a estas informaçóes e avalia especificamente estas 6 dimensões.

O IMPRAFE-54 foi construído conforme os pressupostos da Teoria da Autodeterminação (TAD) ${ }^{5,6}$. Esta Teoria é composta por um conjunto de 6 miniteorias, sendo uma delas específica aos conteúdos das metas. Sistematizada por DECI E RYAN ${ }^{5,6}$, a TAD é amplamente aceita e utilizada em diversas áreas do conhecimento: educação ${ }^{7,8}$, saúde ${ }^{9,10}$, administração de empresas ${ }^{11,12}$, ambientalismo ${ }^{13,14}$, religião ${ }^{15,16}$, entre outros, inclusive no esporte e atividade física ${ }^{17,18}$. Esta teoria preconiza que um sujeito pode ser motivado em diferentes níveis (intrínseca ou extrinsecamente) ou, ainda, estar amotivado à prática de qualquer atividade.

Quando intrinsecamente motivado, o sujeito 
ingressa na atividade por vontade própria, diga-se, pelo prazer e satisfação do processo de conhecê-la, explorála, aprofundá-la. Comportamentos intrinsecamente motivados são comumente associados com bemestar psicológico, interesse, alegria e persistência ${ }^{6}$. A motivação tem sido subdividida em três tipos: para saber, para realizar e para experiência. Já a motivação extrínseca, segundo RyAn E DECI ${ }^{19}$, ocorre quando uma atividade é motivada por expectativas de resultados ou contingências não inerentes à própria atividade. Entretanto, esses motivos podem variar grandemente em relação ao seu grau de autonomia, criando, basicamente, três categorias dessa motivação: regulação externa, regulação interiorizada, regulação identificada. Ryan e DeCI ${ }^{19}$, também citam a amotivação, construção motivacional presente em indivíduos que não conseguem identificar um motivo para realizar alguma atividade física ou simplesmente não se sentem capazes de realizá-la. Segundo esses indivíduos, tal atividade não lhes trará nenhum benefício ou não conseguirão realizá-la de modo satisfatório $^{20}$.

As medidas são fundamentais no estudo da motivação. Diferentes instrumentos têm sido usados para medir este construto. No âmbito internacional, um importante instrumento foi desenvolvido por Gill, Gross e Huddleston ${ }^{21}$ com a construção do Participation Motivation Inventory (PMI-30). O PMI-30 mede oito dimensōes da motivação. Embora este inventário apresente consistência interna insatisfatória $(\alpha<0,70)$ em cinco das suas oito dimensōes, foi largamente utilizado em vários estudos nos $\mathrm{EUA}^{22,23,24}$, muitos deles feitos com adolescentes ${ }^{25,26,27}$, e teve uma versão traduzida para o espanhol ${ }^{28}$. Outro importante instrumento é o Intrinsic Motivation Inventory (IMI). Esta escala tem sido utilizada em diferentes estudos ${ }^{29,30,31}$ e a sua validade foi novamente discutida e confirmada em $1995^{32}$. Além disso, uma versão portuguesa foi desenvolvida e validada em 2001, a IMIp ${ }^{33}$. Existe ainda, na verdade, um grande número de escalas, tais como o Exercise Motivation Inventory (EMI) ${ }^{34,35}$, o qual foi utilizado em estudantes universitários ${ }^{36,37}$ e em adultos jovens ${ }^{38}$; a Sport Motivation Scales $(\mathrm{SMS})^{39}$ - as quais tiveram versões traduzidas para o português $s^{40} \mathrm{e}$ uma validação preliminar de sua versão em italiano ${ }^{41}$. Sua concepção original foi revisada para a SMS-II ${ }^{42}$ e, depois, reeditada para uma nova, com seis fatores ${ }^{43}$; a L'Échelle de Motivation pour les Sports $\left(\right.$ ÉMS $^{20}$. No âmbito nacional, alguns estudos têm procurado desenvolver escalas adequadas à cultura local (que não sejam simples traduções das escalas internacionais) e que tenham propriedades métricas que recomendem sua utilização. Um esforço neste sentido deu origem ao Inventário de Motivação para a Prática Desportiva (IMPD) ${ }^{44}$. Analisando especificamente um dos motivos à prática esportiva, existe a Escala Balbinotti de Motivos à Competitividade no Esporte (EBMCE-18) ${ }^{45}$, validada por BALBINOTTI et $\mathrm{al}^{46}$. Por fim, foi criado o Inventário de Motivação para a Prática Regular de Atividade Física e/ou Esportiva (IMPRAFE) ${ }^{47}$, que propõe investigar seis dimensões motivacionais. Após a criação uma primeira versão, com 120 itens, utilizada em estudos preliminares, foi desenvolvida uma segunda versão com 126 itens $^{47}$. Este instrumento teve suas propriedades métricas verificadas e apresentou índices adequados de consistência interna para cada dimensão ${ }^{48}$. Posteriormente foram desenvolvidas outras duas versões: uma com 132 iten s $^{49}$ e uma versão reduzida, com 54 itens ${ }^{4}$. Estes instrumentos têm sido amplamente aplicados no Brasil em esportes como a ginástica rítmica ${ }^{50}$, tênis ${ }^{51}$, basquetebol ${ }^{52}$ e corrida de rua $^{53,54}$, bem como em frequentadores de academias de ginástica ${ }^{55}$, entre outros.

$\mathrm{O}$ conhecimento dos motivos pelos quais um sujeito possa vir a praticar uma determinada atividade física ou esportiva pode, quando adequadamente utilizado, aumentar as possibilidades de ingresso e permanência de indivíduos nesta prática ${ }^{56,57,58,53}$. Segundo diversos autores ${ }^{59-62}$, a forma mais objetiva e sistemática de acessar este tipo de informação é com o uso de questionários, inventários e/ou escalas métricas de construtos psicossociais. Daí a importância de avaliar as propriedades métricas do IMPRAFE- 54 . Por este motivo, e a partir dos aspectos teóricos e empíricos supramencionados, o presente estudo teve por objetivo apresentar novas evidências de validade e precisão do Inventário de Motivos à Prática Regular de Atividade Física e/ou Esporte (IMPRAFE-54) ${ }^{4}$ em uma amostra de atletas infantojuvenis, federados, de basquetebol participantes de competições institucionalizadas a nível nacional. Para tanto, especificamente, foi estimada a estrutura interna relativa ao construto segundo os dados disponíveis; foi testada a estabilidade da estrutura interna obtida com os dados disponíveis; e, por fim, foi estimada a consistência interna das dimensões avaliadas pelo IMPRAFE-54 ${ }^{4}$. Justifica-se a realização deste estudo pela necessidade de se confirmar a estrutura original do referido instrumento em diferentes contextos. $\mathrm{O}$ acúmulo de estudos desta natureza reforça a ideia de que o IMPRAFE-54 é válido para diferentes amostras (ambientes). 


\section{Método}

\section{Sujeitos}

Este estudo contou com a participação de 438 jovens atletas de basquetebol federados de ambos os sexos, sendo 275 do sexo masculino $(62,8 \%)$ e 163 do sexo feminino $(37,2 \%)$, com idades variando de 13 a 16 anos, que participam de Competiçóes Institucionalizadas (Campeonatos Escolares, Campeonatos Inter-Clubes, JERGS, etc.), vinculados a instituiçóes esportivas dos estados do Rio Grande do Sul e Santa Catarina. A amostra foi escolhida de acordo com a disponibilidade dos jovens e a acessibilidade nas instituiçóes. De acordo com Maguire e Rogers ${ }^{63}$, trata-se de uma amostra não-aleatória recomendada para estudos em educação e psicologia. Costello e Osborne ${ }^{64}$ afirmam que a maioria $(62,9 \%)$ das análises fatoriais têm sido realizadas com 10 sujeitos por item, ou menos, sendo, ainda, que um número importante de estudos tem sido realizado com dois sujeitos por item. Ainda afirmam que a grande maioria dos estudos $(63,9 \%)$ tem adotado um tamanho amostral maior que cinco e menor ou igual a 20, e que os resultados com este tamanho amostral têm se apresentado satisfatórios (especialmente quanto maior a amostra) ${ }^{64}$. Portanto, acredita-se que o tamanho amostral obtido é suficiente para tais análises, especialmente se levado em consideração a especificidade da população.

\section{Instrumentos}

Os jovens participantes responderam dois instrumentos: o Questionário de Identificação e Controle de Variável (QICV) (para controle das variáveis sexo e idade) e Inventário de Motivos à Prática Regular de Atividades Físicas e/ou Esportivas (IMPRAFE-54) ${ }^{4}$. Como mencionado, o Inventário de Motivos Para a Prática Regular de Atividades Físicas e/ou Esportivas (IMPRAFE-54) ${ }^{4}$ foi elaborado com base nos 126 itens do IMPRAF-126 ${ }^{47}$. Neste inventário, foram selecionados os 54 itens, que melhor representavam cada uma das dimensões propostas pela teoria. Sendo assim, cada dimensão possui 9 itens visando aparar todas as arestas do construto - os motivos. O número reduzido de itens se justifica para tornar o instrumento menos cansativo para os respondentes e, consequentemente, menos repetitivo, facilitando a aplicação para grandes amostras. Os itens foram agrupados 6 a 6 , seguindo a sequência das dimensões mais frequentemente mencionadas na literatura ${ }^{2}$ : Controle de Estresse (ex.: liberar tensões mentais), Saúde (ex.: manter a forma física), Sociabilidade (ex.: estar com amigos), Competitividade (ex.: vencer competiçóes), Estética (ex.: manter bom aspecto) e Prazer (ex.: meu próprio prazer). As respostas aos itens deste inventário devem ser dadas conforme uma escala bidirecional, de tipo Likert, graduada em cinco pontos, indo de "isto me motiva pouquíssimo" (1) a "isto me motiva muitíssimo" (5). O inventário conta ainda com uma escala de verificação que permite a avaliação do nível de atenção do sujeito durante a aplicação. Seis itens, um de cada dimensão, tomados aleatoriamente, são repetidos no final do inventário. A medida da validade da aplicação é obtida através da análise das diferenças entre as respostas.

\section{Procedimentos Éticos}

O Comitê de Ética em Pesquisa da Universidade Federal do Rio Grande do Sul analisou e aprovou esse projeto de pesquisa sob o número de referência 2007722. Os técnicos ou responsáveis pelos atletas foram contatados para explicar detalhadamente os objetivos da pesquisa. O mesmo foi feito com os atletas. A permissão dos pais e/ou responsáveis foi obtida através do termo de consentimento. Ainda, foi explicado que os participantes poderiam deixar a pesquisa a qualquer momento. Os instrumentos foram aplicados individualmente ou em pequenos grupos, no intervalo das competiçóes de basquetebol. Cada participante teve um tempo de vinte e cinco minutos para respondê-los.

\section{Procedimentos Estatísticos}

Primeiramente, somente a título de verificaçáo e comparação com estudos realizados anteriormente $e^{4,47}$, foi realizada uma estimaçáo da estrutura interna do construto medido segundo os dados disponíveis. Para tanto, foram observados os pré-requisitos relativos à análise fatorial exploratória (cálculo do determinante da matriz de correlação, cálculo Kaiser-MeyerOlkin (KMO), teste de esfericidade de Bartlett e matrizes inversa de antimagem e reproduzida). Com relação à análise fatorial confirmatória, foram oportunamente identificados e removidos 
os casos aberrantes. Por fim, pode-se afirmar que os pré-requisitos aos cálculos alfa de Cronbach e métodos das metades (análise de itens, correlação item-item, correlação item-total, alpha de Cronbach se item retirado) foram igualmente realizados e apresentados. A realização da análise fatorial exploratória e dos cálculos de consistência interna foram realizados com a ajuda do pacote estatístico SPSS 22.0 e a análise fatorial confirmatória com o auxílio do AMOS 22.0.

\section{Resultados e Discussão}

A fim de responder, adequadamente, à questão de pesquisa, procedeu-se à exploraçáo dos escores obtidos pelo IMPRAFE-54, segundo princípios norteadores comumente aceitos na literatura especializada ${ }^{61,65,66}$. Os resultados obtidos através da análise geral dos itens, da estimação da estrutura interna, da análise fatorial confirmatória, e dos cálculos de consistência interna serão apresentados de forma sucessiva e sistemática.

Destaca-se que a apresentação formal e inicial da análise geral dos itens, neste estudo, tem por objetivo demonstrar a confiabilidade dos valores das médias observadas, pois estas podem sofrer influência negativa pela presença de casos extremos e, portanto, podem não ser representativas dos comportamentos inventariados, diminuindo, assim, o valor das conclusóes ${ }^{61,65,64}$.

\section{Análise geral dos itens e estimaçáo da estrutura interna do construto}

Destaca-se que as médias encontradas para cada um dos 54 itens estudados individualmente variaram entre 2,26 e 4,40; com desvios-padróes associados variando entre 0,946 a 1,544. Esta variabilidade dos resultados demonstra adequada homogeneidade na dispersão avaliada, pois o valor do desvio padrão, independente do item, é menor do que a metade do valor da média. Esses resultados indicam que, em média, os jovens respondem aos itens do IMPRAFE-54 de forma um pouco mais positiva que negativa. Duas interpretaçóes de caráter positivo são possíveis: a) não houve aderência predominante (seja positiva ou negativa) a nenhum dos itens isolados, ou seja, itens com médias semelhantes aos valores extremos (1 ou 5), o que poderia indicar ausência de variabilidade de respostas - condição que impediria o prosseguimento das análises; e b) podia-se esperar tal fenômeno, pois todos os sujeitos avaliados são praticantes regulares de atividades esportivas, portanto a variável (ou dimensão) motivos para a prática regular de atividades esportivas deve ser uma faceta comportamental importante e, desta forma, supóe-se ocupar certo destaque na dinâmica de suas personalidades.

Já a média encontrada para o instrumento total foi de 193,01 com um desvio-padrão associado de 33,78 . Posto que, o intervalo total esperado era de 54 a 270 pontos (com média esperada de 162 pontos) e o observado foi, efetivamente, de 88 a 270 (com um intervalo interquartil de 53,25 pontos), observamse valores próximos entre as médias esperada e observada. A mediana das correlaçóes item-item foi fraca $(r=0,24)$, possivelmente em função de estar se medindo em um mesmo instrumento seis dimensóes. Além disso, nenhum item revelou correlações com valores inferiores a - 0,06 (correlação nula) ou superiores a 0,79 (correlação forte), ou seja, todos os itens estão medindo um mesmo conceito, não havendo relação negativa entre eles, e todos os itens estão avaliando diferentes facetas deste conceito, não havendo correlação perfeita entre eles. A mediana das correlaçóes item-total foi desejável $(r=0,49)$ e nenhum item revelou correlaçóes com a escala total inferior a 0,29. Esses resultados reforçam a interpretação de que os itens são adequados e pertinentes.

As correlaçôes item-item - particularmente aquelas inferiores a 0,25 - poderiam indicar certa necessidade de revisão de suas qualidades métricas, quando considerados isoladamente. Entretanto, ao se comparar os índices Alpha, que variaram de 0,942 a 0,944, para os itens retirados (excluídos), com o coeficiente Alpha para a escala total sem a exclusão de nenhum item $(0,944)$, verifica-se que esse procedimento é desnecessário e improdutivo, pois pode causar perda na validade de conteúdo, principalmente quanto à pertinência e à adequada exploração de todas as arestas - significados, nuances e interpretaçôes - do conceito. Finalmente, destacase que o valor Alpha encontrado para a escala total revela um escore desejável da consistência interna do instrumento completo. Trata-se de um importante preditor da confiabilidade e precisão 
desses resultados.

O QUADRO 1 demonstra, graficamente, que não houve distorção importante na curva de distribuição dos resultados do IMPRAFE-54 (ver histograma). Os pontos dos valores observados mantiveram-se muito próximos à reta dos valores esperados (ver gráfico Q-Q) e, portanto, não houve a presença de valores extremos (casos aberrantes) que pudessem distorcer os resultados (ver gráfico de bigodes), caracterizando a adequação do uso de instrumentais paramétricos de avaliação. Todos esses resultados sustentam a adequabilidade dos dados em estudo e a continuidade das análises.

QUADRO 1 - Demonstração gráfica da aderência à normalidade dos dados em estudo (n=438).

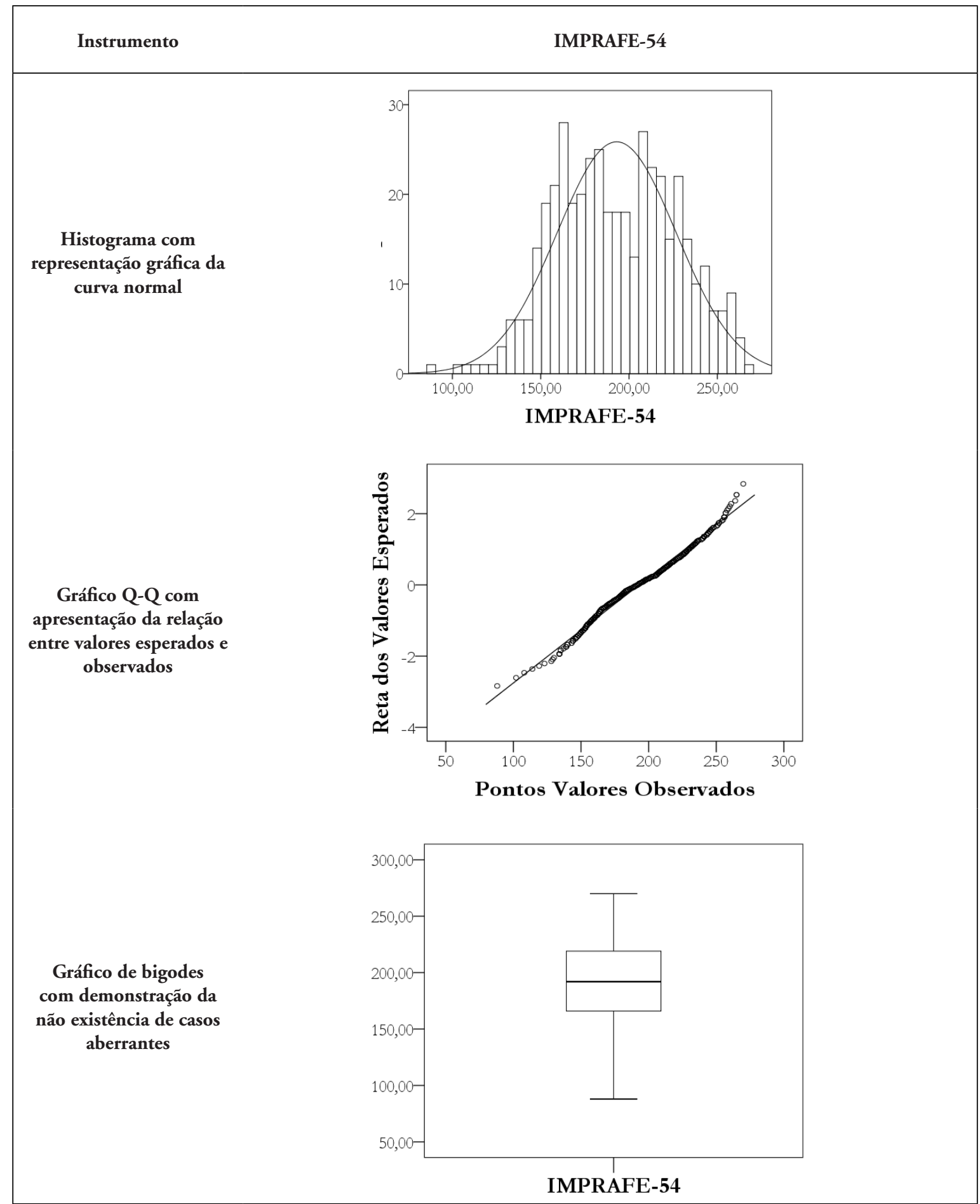


Feito isso, a fim de se garantir a adequada interpretação da estimação da estrutura interna do construto, foi estimado o coeficiente Kaiser-MeyerOlkin $(\mathrm{KMO}=0,92)$, foi calculado o Determinante de Matriz de Correlaçáo (1,004E-013) e aplicado o teste de esfericidade de Bartlett $(\mathrm{p}<0,001)$. Seus respectivos valores indicam que as correlaçóes entre os itens são suficientes - e mesmo muito adequadas - para o prosseguimento das análises. Ainda, o resultado da medida de redundância de informação $(|\mathrm{R}|$ diferente de 0$)$ indica a ausência de todo tipo de repetiçáo das ligaçóes correlativas lineares. Todos esses dados asseguram a pertinência dos cálculos fatoriais ${ }^{67}$.

Após uma análise ULS (Unweighted Least Squares), seguida de rotação oblíqua (com delta de 0), verificou-se, por meio do gráfico "scree" (ver FIGURA 1), uma solução fatorial em seis fatores, corroborando com o modelo proposto pelo autor e com os estudos realizados anteriormente ${ }^{47,48}$. Esta solução fatorial explica $58,43 \%$ da variância total do construto medido - valor superior ao limite do desejável ( $>50 \%$ ) de acordo com a literatura ${ }^{6,16}$. A variância total explicada neste estudo é compatível com aquelas encontradas em outros estudos que avaliaram instrumentos de avaliação de motivos à prática regular de atividade física, nos quais a variância total explicada foi de 54\% para o inventário IMIp ${ }^{48}, 69.40 \%$ para o $\mathrm{EMI}^{49} \mathrm{e}$ $71.75 \%$ para o SMS-II ${ }^{58}$. Sendo assim, as análises subsequentes foram realizadas restringindo o número de fatores em seis.

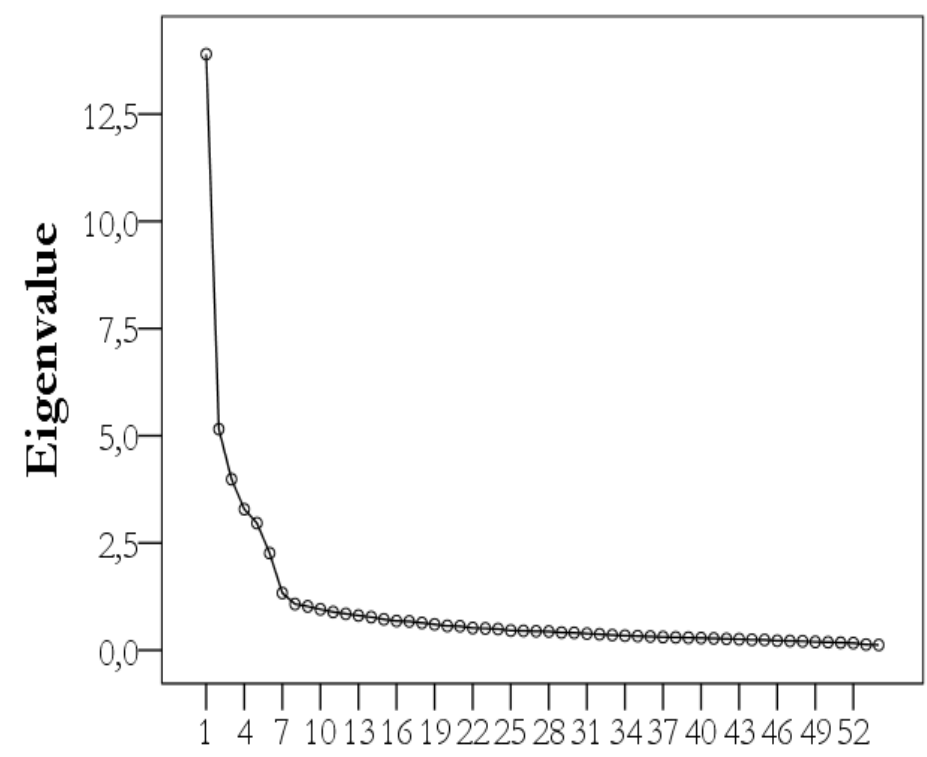

Número de componente

FIGURA 1 - Gráfico "Scree" sugerindo a solução em seis fatores.

Ainda, destaca-se que as comunalidades dos itens são todas superiores a 0,41 ; que trata-se de uma solução pura, ou seja, não houve dupla saturação significativa em qualquer dos itens medidos; e os itens saturaram de forma importante (|Satf $\mid>0,482$ ) em seus próprios fatores de origem. Este resultado é semelhante àqueles encontrados na literatura ${ }^{2,68}$, permitindo a mesma nomeação dos fatores identificados (Controle de Estresse, Saúde, Sociabilidade, Competitividade, Estética, Prazer).

Os motivos que levam os indivíduos à prática de atividades físicas e/ou esportiva, conforme medida pelo IMPRAFE-54, não é de natureza unidimensional. Portanto, não se pode, ou não seria prudente interpretar esta variável em um sentido geral, tal como: "este jovem atleta é motivado". Observaçôes/interpretaçôes como esta podem, no mínimo, representar uma importante falta de precisão. Parece, considerando estes resultados, que seria mais adequado especificar o motivo do qual se está falando.

Ao classificar alguém como motivado é necessário especificar a que e pelo que. Sendo assim, é imprescindível identificar qual ou quais razôes (motivos) pelas quais essa pessoa está engajada neste comportamento específico. Outra possível alternativa para classificar alguém como motivado é a de avaliar em que nível este sujeito é autodeterminado. Neste caso, é necessária a investigação e aplicação de instrumentos que 
sejam específicos e precisos ao medir estes conteúdos. Neste sentido, o Inventário de Autodeterminação para Praticantes de Atividades Esportivas ${ }^{68}$, o qual também é baseado na Teoria da Autodeterminaçãoo, investiga o quão autodeterminado - ou motivado - o indivíduo é. Dentro do âmbito da motivação, o estudo dos motivos é essencial e este é medido pelo IMPRAFE-54.

Segundo a teoria e diversos estudos, um indivíduo pode ser igualmente motivado por dois ou mais motivos ${ }^{1,50,69}$. No entanto, algumas dimensôes avaliadas pelo IMPRAFE-54 indicam uma maior tendência à motivação intrínseca. $\mathrm{O}$ principal exemplo é a dimensão Prazer. Portanto, o conhecimento dos motivos que levam indivíduos à prática esportiva pode apresentar indicações importantes no sentido da identificação do quão motivado o indivíduo é.

Saber os motivos pelos quais pessoas procuram determinadas atividades físicas ou esportivas é de extrema importância, visto que pode, quando adequadamente utilizado, aumenta a probabilidade de ingresso e permanência dos indivíduos nessa prática ${ }^{56,57,58,53}$. Dessa forma, o indivíduo poderá desfrutar por mais tempo os benefícios promovidos pela modalidade esportiva escolhida. Neste sentido, pode-se questionar modelos de treinamento predeterminados, afinal é recomendável que o treinamento de alguém motivado pela Sociabilidade seja diferente daquele motivado pela Competitividade, por exemplo.

\section{Análise fatorial confirmatória}

Visto que o modelo hexadimensional anteriormente teorizado e testado se confirmou ${ }^{2}$ e que os itens que compõem o IMPRAFE-54 saturam nas dimensóes que teoricamente deveriam saturar, cabe agora testar se os dados disponíveis se ajustam ao modelo teórico (hexadimensional) proposto. Para tanto, uma análise fatorial confirmatória ULS (Unweighted Least Squares) foi calculada e seus resultados $\left(\chi^{2}=4723,807 ; \mathrm{gl}=1362 ; \mathrm{p}<0,001 ; \chi^{2} / \mathrm{gl}=\right.$ 3,468; GFI $=0,970 ;$ AGFI $=0,967 ;$ SRMR $=$ 0,0574 ; NFI $=0,962$ ) podem ser considerados adequados/aceitáveis $^{61,70}$. Na verdade, o único resultado insatisfatório é o da significância do $\chi^{2}$. Resultados insatisfatórios, como estes, são tipicamente encontrados em grandes amostras.
Por essa razão, alguns autores ${ }^{71,72}$ têm descartado a significância do $\chi^{2}$ de suas análises. Sendo assim, a validade do modelo hexagonal do instrumento é apoiada pelos dados empíricos disponíveis. Outros estudos que avaliaram a validade fatorial confirmatória de instrumentos, tais como o IMIp ${ }^{33}$ e o SMS-II ${ }^{73}$, encontraram indicadores muito semelhantes e um $\chi^{2}$ também significativo. Numa interpretação de caráter mais geral desses dados, pode-se inferir que o funcionamento cognitivo do grupo de atletas pesquisados, quando requeridos a responder sobre os motivos que os levam a praticar regularmente atividades físicas ou esportivas, pode ser compartimentalizado em seis dimensões distintas. Destaca-se, então, que esse modelo hexadimensional foi confirmado.

\section{Cálculos de consistência interna}

A precisão da medida de cada uma das 6 dimensôes do construto "Motivos", pode ser respondida pelo viés da consistência interna (Alpha de Cronbach e cálculo pelo Método das Metades). Os resultados dos cálculos Alpha de Cronbach, por dimensão em estudo, estão descritos na TABELA 1 , e variam de 0,86 a 0,94 - índices semelhantes aos encontrados por BARBOSA ${ }^{2}$, que variaram de 0,82 a 0,94 . E, ligeiramente superior ao encontrado em estudos que avaliaram a consistência interna de outros instrumentos, tais como o IMPD ${ }^{44}$, o PMI$30^{21}$, o $\mathrm{EMI}^{34}$ e o SMS-II ${ }^{73}$. Tais resultados são excelentes indicadores de precisão de cada uma das seis dimensões medidas ${ }^{66}$, podendo-se dizer que os resultados obtidos em cada um dos nove itens de cada dimensão são consistentes entre si, representando uma medida precisa do construto de primeira ordem.

Afim de aprofundar o estudo da consistência interna, foi realizado o cálculo desta pelo Método das Metades. A TABELA 1 apresenta os resultados obtidos, através dos coeficientes de Guttman e de Spearman-Brown, que confirmam satisfatoriamente a interpretação de que cada uma das dimensões em análise (CE, Sa, So, Co, Es e Pr) avalia, com precisão, o construto de primeira ordem testado. Além disso, analisando os coeficientes Alpha de Cronbach para cada uma das duas metades de cada orientação, pode-se perceber a importância de se manter o número de itens proposto (nove, por cada dimensão). 
Saldanha RP, et al.

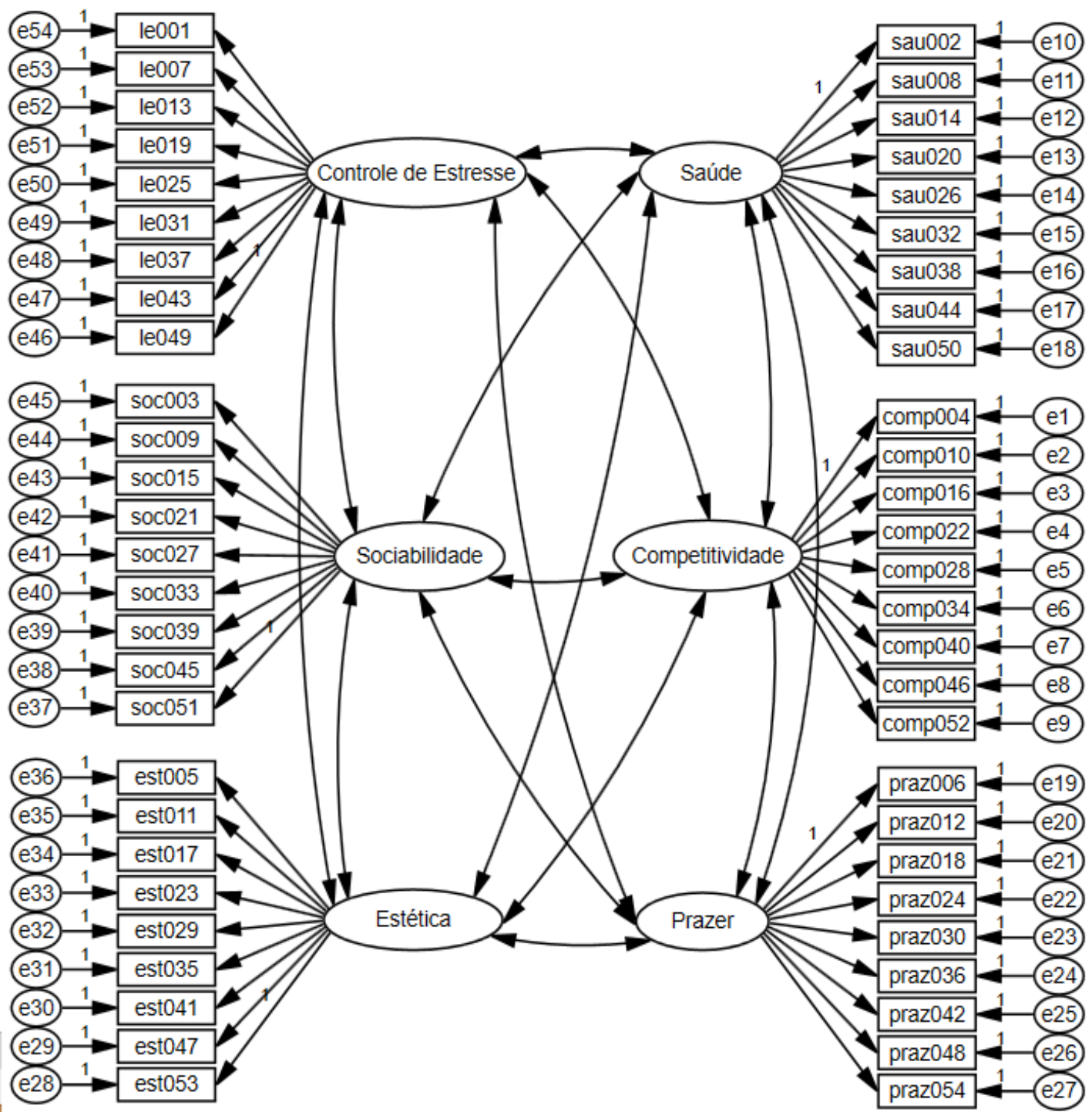

FIGURA 2 - Modelo teórico estrutural de avaliação dos motivos à prática regular de atividades físicas e esportivas.

TABELA 1 - Coeficientes relativos ao Método das Metades.

\begin{tabular}{|c|c|c|c|c|c|c|}
\hline \multirow{2}{*}{ Dimensão } & \multicolumn{3}{|c|}{ Alpha de Cronbach } & \multirow{2}{*}{$\begin{array}{c}\text { Correlação } \\
\text { entre } \\
\text { Metades }\end{array}$} & \multirow{2}{*}{$\begin{array}{l}\text { Coeficiente } \\
\text { S-Bcorrigido }\end{array}$} & \multirow{2}{*}{$\begin{array}{l}\text { Coeficiente } \\
\text { de Guttman }\end{array}$} \\
\hline & Total & Metade 1 & Metade 2 & & & \\
\hline $\mathrm{CE}$ & 0,905 & 0,834 & 0,832 & 0,80 & 0,89 & 0,888 \\
\hline Sa & 0,886 & 0,794 & 0,797 & 0,80 & 0,89 & 0,891 \\
\hline So & 0,922 & 0,855 & 0,855 & 0,867 & 0,93 & 0,918 \\
\hline Co & 0,881 & 0,779 & 0,780 & 0,832 & 0,909 & 0,893 \\
\hline Es & 0,939 & 0,881 & 0,889 & 0,892 & 0,944 & 0,937 \\
\hline $\operatorname{Pr}$ & 0,860 & 0,742 & 0,767 & 0,773 & 0,873 & 0,859 \\
\hline
\end{tabular}


Sendo assim, pode se afirmar que este estudo apresentou resultados que indicam a possibilidade de aplicação fidedigna de um instrumento de medida da motivação em esportistas. A partir dos resultados obtidos, podese afirmar que o IMPRAFE-54 é perfeitamente aplicável a uma população específica, no caso atletas de basquetebol participantes de competições institucionalizadas. Especificamente respondendo aos objetivos do estudo, pode-se afirmar que o IMPRAFE-54 realmente apresenta 6 dimensões, as quais se adequam bem ao modelo teorizado inicialmente. Além disso, tanto as dimensões quanto o instrumento possuem resultados de consistência interna elevados, podendo-se ressaltar a confiabilidade dos resultados obtidos com o uso desta ferramenta.

Este achado é de grande valor, visto que inicialmente este instrumento foi validado para uma amostra geral de esportistas ${ }^{2}$. Isto significa que, além deste instrumento possuir uma alta validade externa (ou seja, ser aplicável a uma amostra geral), possui uma forte validade interna também (é também aplicável a populaçôes e esportes específicos, no caso jogadores de basquetebol infantojuvenis). Este resultado reforça as evidências de validade deste instrumento, o qual vem sendo amplamente utilizado em pesquisas relacionadas à psicologia do esporte. Destaca-se ainda a importância da constante e sistemática apresentação de evidências de instrumentos psicométricos, ressaltando que esta é uma das formas mais indicadas para se conferir credibilidade aos estudos empíricos.

Por fim, estes resultados podem ser particularmente úteis para treinadores e psicólogos do esporte, visto que a partir da análise dos motivos que levam estes indivíduos à prática do basquete, podem planejar de forma mais adequada seus treinamentos e intervenções. Contudo, outras medidas de avaliação podem ser interessantes num sentido de complementar as informaçôes já obtidas e extrapolar a um contexto maior.

\section{Abstract}

(Falta título em inglês)

The main question of this study was if it can be found indexes of exploratory and confirmatory factorial validity and reliability suitable to use the Inventory of Reasons to Regular Physical Activity and/or Sports Practice (IMPRAFE-54) with junior basketball players. A sample of 438 young basketball players of both sexes, aged from 13 to 16, tied to sports institutions participated in the study. Two instruments were answered: Identification Questionnaire and Variable Control (QIVC) and the Inventory of Reasons to Regular Physical Activity and/or Sports Practice (IMPRAFE-54). Exploratory factorial analysis ULS (Unweighted Least Squares), followed by oblique rotation (with 0 delta), identified six factors measured by the instrument, explaining 58.43\% of the measured construct's total variance (six dimensional factor solution). It indicates that this is a valid and reliable inventory, as the six dimensions had Cronbach's alpha indices greater than 0.86 . To test if the exploratory model is confirmed, a confirmatory factor analysis ULS (Unweighted Least Squares) was calculated and its results $\left(X^{2}=4723.807 ; d f=1362, p<0.001 ; X^{2} / g l=3,468 ; G F I=0.970\right.$, $\mathrm{AGFI}=0.967 ; \mathrm{SRMR}=0.0574 ; \mathrm{NFI}=0.962$ ) were acceptable. This study indicates that the IMPRAFE-54 is applicable to a specific population, in this case to junior competitive basketball athletes. These results can be particularly useful for coaches and sport psychologists, since based on the analysis of the reasons that these individuals practice basketball, they can plan more accurately their trainings and interventions.

KEYWORDS: Reasons; Psychometrics; Validation; Sport.

\section{Referências}

1. Vansteenkiste M, Niemiec CP, Soenens B. The development of the five mini-theories of self-determination theory: an historical overview, emerging trends, and future directions. In: The Decade Ahead: Theoretical Perspectives on 
Motivation and Achievement. Bingley: Emerald Group Publishing Limited; 2010. p.105-165.

2. Barbosa MLL. Propriedades métricas do inventário de motivação à prática regular de atividade física (IMPRAF-126) [dissertação]. Porto Alegre (RS): Universidade Federal do Rio Grande do Sul, Escola de Educação Física; 2006.

3. Saldanha RP. Motivação à prática regular de atividades físicas: um estudo com atletas de basquetebol infant-juvenis (13 a 16 anos) [dissertação]. Porto Alegre (RS): Universidade Federal do Rio Grande do Sul, Escola de Educação Física; 2008.

4. Barbosa MLL, Balbinotti MAA. Inventário de Motivos à Prática Regular de Atividade Física e/ou Esportiva (IMPRAFE-54). Porto Alegre (RS): Universidade Federal do Rio Grande do Sul; 2006.

5. Deci EL, Ryan RM. Intrinsic motivation and self-determination in human behavior. Nova Iorque: Plenum; 1985.

6. Ryan RM, Deci EL. Self-Determination Theory and the facilitation of intrinsic motivation, social development, and well-being. Am Psychol. 2000;55(1):68-78.

Anastasi A, Urbina S. Testagem Psicológica. Porto Alegre: Artes Médicas, 2000.

7. Haerens L, Aelterman N, Van den Berghe L, De Meyer J, Soenens B, Vansteenkiste M. Observing physical education teachers' need-supportive interactions in classroom settings. J Sport Exercise Psy. 2013;35:3-17.

8. Wilkesmann U, Schmid CJ. Intrinsic and internalized modes of teachingmotivation. Evid-based HRM. 2014;2:6-27.

9. Chan DKC, Fung YK, Xing S, Hagger MS. Myopia prevention, near work, and visual acuity of college students: Integrating the theory of planned behavior and self- determination theory. J Behav Med. 2014;37:369-380.

10. Hagger MS, Hardcastle SJ, Chater A, Mallett C, Pal S, Chatzisarantins NLD. Autonomous and controlled motivational regulations for multiple health-related behaviors: between- and within-participants analyses. Health Psychol Behav Med. 2014;2:565-601.

11. Fernet C, Austin S, Trépanier, SG, Dussault M. How do job characteristics contribute to burnout? Exploring the distinct mediating roles of perceived autonomy, competence, and relatedness. Eur J Work Organ Psy. 2013;22:123-137.

12. Trépanier SG, Fernet C, Austin S. Workplace psychological harassment among Canadian nurses: A descriptive study. J Health Psychol. 2013;18:383-396.

13. Lavergne KJ, Sharp EC, Pelletier LG, Holtby A. The role of perceived government style in the facilitation of selfdetermined and non self-determined pro-environmental behavior. J Environ Psychol. 2010;30:169-177.

14. Sheldon KM, Nichols CP, Kasser T. Americans recommend smaller ecological footprints when reminded of intrinsic American values of self-expression, family, and generosity. Ecopsychol. 2011;3:97-104.

15. Martos T, Kezdy A, Horvath-Szabo K. Religious motivations for everyday goals: Their religious context and potential consequences. Motiv Emotion. 2011;35:75-88.

16. Soenens B, Neyrinck B, Vansteenkiste M, Dezutter J, Hutsebaut D, Duriez B. How do perceptions of God as autonomysupportive or controlling relate to individuals' social-cognitive processing of religious contents? The role of motives for religious behavior. Int J Psychol Relig. 2012;22:1-21.

17. Slingerland M, Haerens L, Cardon G, Borghouts L. Differences in perceived competence and physical activity levels during single-gender modified basketball game play in middle school physical education. Eur Phys Educ Rev. 2014;20:20-35.

18. Van de Berghe L, Vansteenkiste M, Cardon G, Kirk D, Haerens L. Research on self-determination in physical education: Key findings and proposals for future research. Phys Educ Sport Pedagog. 2014;1:97-121.

19. Ryan RM, Deci EL. Intrinsic and Extrinsic Motivations: Classic Definitions and New Directions. Contemp Educ Psychol. 2000;25(1):54-67.

20. Brière NM, Vallerand RJ, Blais MR, Pelletier LG. Developpement et validation d'une mesure de motivation intrinséque, extrinséque et d'amotivation en contexte sportif: l'echelle de motivation dans les sports. Int J Sport Psychol. 1995; 26(4):465-489.

21. Gill DL, Gross JB, Huddleston S. Participation motivation in youth sports. Int J Sport Psychol. 1983;14:1-14.

22. Gould D, Feltz D, Weiss M. Motives for patiticipating in competitive youth swimming. Int J Sport Psychol. 1985;16:126140.

23. Klint KA, Weiss MR. Perceived competence and motives for participation in youth sports: a test of Harter's competence motivation theory. J Sport Psychol. 1987;9:55-65.

24. Brodkin P, Weiss M. Developmental differences in motivation for participating in competitive swimming. JSEP. 1990;12:248-263.

25. Salguero A, Gonzalez-Boto R, Tuero C, Marquez S. Relationship between perceived physical ability and sport participation motives in young competitive swimmers. J Sports Med Phys Fitness. 2004;44(3):294-299. 
26. Sit CHP, Lindner KJ. Situational state balances and participation motivation in youth sport: A reversal theory perspective. Brit J Educ Psychol. 2006;76:396-384.

27. Sit CHP, Lindner KJ. Achievement goal profiles, perceived ability and participation motivation for sport and physical activity. Int J Sport Psychol. 2007;38(3):283-303.

28. Salguero A, Gonzalez-Boto R, Tuero C, Marquez S. Development of a spanish version of the participation motivation inventory for young competitive swimmers. Percept Mot Skills. 2003;96(2):637-46.

29. Hassandra M, Goudas M, Chroni S. Examining factors associated with intrinsic motivation in physical education: a qualitative approach. Psychol Sport Exerc. 2003;4:211-223.

30. Koka A, Hein V. Perceptions of teacher's feedback and learning environment as predictors of intrinsic motivation in physical education. Psychol Sport Exerc. 2003;4(4):333-346.

31. Tas C, Brown EC, Esen-Danaci A, Lysaker PH, Brüne M. Intrinsic motivation and metacognition as predictors of learning potential in patients with remitted schizophrenia. J Psychiat Res. 2012;46(8):1086-1092.

32. Duda JL, Chi L, Newton M, Walling MD, Catley D. Task and ego orientation and intrinsic motivation in sport. Int J Sport Psychol. 1995;26(1):81-97.

33. Fonseca AM, Brito AP. Propriedades psicométricas da versão portuguesa do Intrinsic Motivation Inventory (IMIp) em contextos de actividade física e desportiva. Anál. psicol. 2001;1(XIX): 59-76.

34. Markland D, Hardy L. The exercise motivation inventory: Preliminary development and validity of a measure of individuals' reasons for participation in regular physical exercise. Pers Indiv Differ. 1993;15:289-296.

35. Markland D. Ingledew DK. The measurement of exercise motives: Factorial validity and invariance across gender of a revised Exercise Motivations Inventory. Brit J Health Psych. 1997;2:361-376.

36. Egli T, Bland HW, Melton BF, Czech DR. Influence of Age, Sex, and Race on College Students' Exercise Motivation of Physical Activity. J Am Coll Health. 2011;59(5):399-406.

37. Pope L, Harvey J. The impact of incentives on intrinsic and extrinsic motives for fitness-center attendance in college first-year students. Am J Health Promot. 2015;29(3):192-9.

38. Ingledew DK, Markland D, Ferguson E. Three Levels of Exercise Motivation. Appl Psychol Health Well-Being. 2009;1(3):336-355.

39. Butt DS. Psychology of sport: The behavior, motivation, personality, and performance of athletes. Nova Iorque: Van Nostrand Reinhold; 1987.

40. Bara FM, Andrade D, Miranda R, Núñez JL, Martín-Albó J, Ribas PR. Preliminary validation of a brazilian version of the sport motivation scale. Univ Psychol. 2011;10(2):557-566.

41. Candela F, Zucchetti G, Villosio C. Preliminary validation of the Italian version of the original sport motivation scale. J Hum Sport Exerc. 2014;9(1):136-147.

42. Pelletier LG, Tuson KM, Fortier MS, Vallerand RJ, Brière NM, Blais MR. Toward a New Measure of Intrinsic Motivation, Extrinsic Motivation, and Amotivation in Sports: The Sport Motivation Scale (SMS). J Sport Exercise Psy. 1995; 17:35-53.

43. Mallett C, Kawabata M, Newcombe P, Otero-Forero A, Jackson S. Sport motivation scale-6 (SMS-6): A revised sixfactor sport motivation scale. Psychol Sport Exerc. 2007;8(5):600-614.

(43)McAuley E, Duncan TA, Tammen VV. Psychometric properties of the Intrinsic Motivation Inventory in a competitive sport setting: A confirmatory factor analysis. Res Q Exercise Sport, 1989;60:48-58.

44. Gaya A, Cardoso M. Os fatores motivacionais para a prática desportiva e suas relaçóes com o sexo, idade e níveis de desempenho desportivo. Rev Perfil. 1998;2(2):40-52.

45. Balbinotti MAA. Escala Balbinotti de Motivos à Competitividade no Esporte. Service d'Intervention et de Recherche en Orientation et Psychologie (SIROP). Montreal: [editora desconhecida]; 2008.

46. Balbinotti MAA, Barbosa MLL, Saldanha R.P, Balbinotti CAA. Estudos fatoriais e de consistência interna da Escala Balbinotti de Motivos à Competitividade no Esporte (EBMCE-18). Motriz: rev educ fís. 2011;17(2):318-327.

47. Balbinotti MAA. Inventário de motivação à prática regular de atividade física e/ou esporte. Porto Alegre: Universidade Federal do Rio Grande do Sul; 2004.

48. Balbinotti MAA, Barbosa MLL. Análise da Consistência Interna e Fatorial Confirmatório do IMPRAFE-126 com praticantes de atividades físicas gaúchos. Psico-USF. 2008;13:1-12.

49. Balbinotti MAA. Inventário de motivação à prática regular de atividade física e/ou esporte. Porto Alegre: Universidade Federal do Rio Grande do Sul; 2009.

50. Fontana PS, Barbosa MLL, Balbinotti MAA, Balbinotti CAA. Estudo das motivaçóes à prática da ginástica rítmica: 
contribuições da pesquisa para o rendimento desportivo. Pensar prát. 2013;16(2):387-400.

51. Balbinotti MAA, Juchem L, Barbosa MLL, Saldanha RP, Balbinotti CAA. Qual o perfil motivacional de tenistas infanto-juvenis brasileiros? Motriz. 2012;18(4):728-734.

52. Balbinotti MAA, Saldanha RP, Balbinotti CAA. Dimensóes motivacionais de basquetebolistas infanto-juvenis: um estudo segundo o sexo. Motriz. 2009;15(2):318-329.

53. Balbinotti MAA, Gonçalves GHT, Klering RT, Wiethaeuper D, Balbinotti CAA. Perfis motivacionais de corredores de rua com diferentes tempos de prática. Rev Bras Ciênc Esporte. 2015;37(1):65-73.

54. Balbinotti MAA, Wiethaeuper D, Gonçalves GHT, Klering RT, Balbinotti CAA. Perfil motivacional de atletas corredores de rua: estudo comparativo segundo o sexo. R Min Educ Fís. 2012;1:2051-2062.

55. Balbinotti MAA, Capozzoli CJ. Motivação à prática regular de atividade física: um estudo exploratório com praticantes em academias de ginástica. Rev Bras Ed Fís Esporte. 2008;22(1):63-80.

56. Allen JB. Social motivation in youth sport. J Sport Exerc Psychol. 2003;25(4):551-567.

57. Costa IT, Samulski DM, Noce F, Costa VT. A importância da atividade física para a manutenção da saúde e os principais fatores que motivam professores, alunos e funcionários de duas universidades brasileiras a praticarem exercícios. Rev Bras Ativ Fís Saúde. 2003;8(1):52-61.

58. Brunet J, Sabiston CM. Exploring motivation for physical activity across the adult lifespan. Psychol Sport Exerc. 2011;12:99-105.

59. Cunha JA. Psicodiagnóstico-R. 5a ed. Porto Alegre: Artes Médicas; 2000.

60. Balbinotti MAA. Vers un modèle explicatif de la cristallisation des préférences professionnelles durant l'adolescence. [Tese] Montreal, Canadá: Universidade de Montreal, Faculdade de Artes e Ciências; 2001.

61. Balbinotti MAA. Para se avaliar o que se espera: reflexóes acerca da validade dos testes psicológicos. Aletheia. 2005;1(21):43-52.

62. Yamaji BHS, Guedes DP. Instrumentos para identificar os motivos para prática de esporte: opçóes disponíveis na literatura. Pensar prát [Internet]. 2015 [citado 24 mai. 2015]; 18(1). Disponível em: http://www.revistas.ufg.br/index. $\mathrm{php/fef/article/view/30015/18332.}$

63. Maguire TO, Rogers WT. Proposed solutions for non randomness in educational research. CJE. 1989;14(2):170-181.

64. Costello AB, Osborne JW. Best Practices in Exploratory Analysis: Four Recommendations fot Getting the Most From Your Analysis. PARE. 2005;10(7):1-9.

65. Bryman A, Cramer D. Quantitative data analysis with IBM SPSS 17, 18 \& 19: a guide for social scientists. New York: Routledge; 2011.

66. Pestana MH, Gageiro JG. Análise de dados para ciências sociais: a complementaridade do SPSS. Lisboa: Silabo; 2014.

67. Dassa C. Analyse multidimensionnelle exploratoire et confirmative. Montreal: Univesité de Montreal; 1999.

68. Balbinotti MAA, Barbosa MLL. Inventário de Autodeterminação para Praticantes de Atividades Esportivas. Service d'Intervention et de Recherche en Orientation et Psychologie (SIROP). Montreal: [editora desconhecida]; 2008.

69. Sampedro LBR, Rocha JM, Klering RT, Saldanha RP, Balbinotti MAA, Balbinotti CAA. Motivaçáo à prática regular de atividades físicas: um estudo com praticantes de Taekwondo. Pensar prát. 2014;17(1):19-32.

70. Xu L, Wang S. Empirical research on construct of chain store logistics capability system. iBusiness. 2012;4:10-17.

71. Cole DA. Utility of confirmatory factor analysis in test validation research. J Consult Clin Psych. 1987; 55:584-594.

72. Marsh HW, Balla JR, McDonald RP. Goodness-of-fit indexes in confirmatory factor analysis: the effect of sample size. Psychol Bull. 1988;103:391-410.

73. Pelletier LG, Rocchi MA, Vallerand RJ, Deci EL, Ryan RM. Validation of the revised sport motivation scale (SMS-II). Psychol Sport Exerc. 2013;14:329-341.

$$
\begin{aligned}
& \text { ENDEREÇO DE CORRESPONDÊNCIA } \\
& \text { Gabriel Henrique Treter Gonçalves } \\
& \text { Rua Jacob Vontobel, 72/202 - Jardim Botânico } \\
& \text { Porto Alegre - RS - BRASIL } \\
& \text { CEP: 90690-080 } \\
& \text { E-mail: gabrielhtg@gmail.com }
\end{aligned}
$$

